\title{
10. Richter und Staatsanwälte
}

Nach der Schilderung charakteristischer Einzelfälle der Hamburger Rechtsprechung in Rassenschandeverfahren bleibt zu fragen, wer für diese Praxis verantwortlich war. Man kommt damit an das Kernproblem der Rechtsgeschichte des Dritten Reiches: Gab es noch richterliche Unabhängigkeit? Wenn ja, wie weit? Konnten die Richter ihrer Rechtsprechungsfunktion noch dienen oder waren sie Erfüllungsgehilfen des nationalsozialistischen Regimes?

Beide Meinungen sind vertreten worden. Apologeten des Dritten Reichs behaupten, in der täglichen Berufsausübung der Richter sei auch während des Dritten Reiches der Grundsatz der Unabhängigkeit der Justiz im großen und ganzen gewahrt geblieben. Richter hingegen behaupten, ihre Unabhängigkeit habe kaum noch zum Schein bestanden, sie seien mehr oder weniger zu Objekten einer gelenkten Justiz geworden.

Beide Erklärungen sind unrichtig. Erstens, weil die Verhältnisse im Laufe der 12 Jahre von 1933 bis 1945 keineswegs konstant waren. Und zweitens, weil diese Fragen nicht einheitlich für die gesamte Rechtspflege gleich beantwortet werden können. Die politischen Gegebenheiten wirkten sich auf den einzelnen Teilgebieten der Rechtsprechung sehr verschieden aus. Je stärker der totalitäre Charakter des Dritten Reiches hervortrat, desto größer wurde die Zahl der Verfahren mit politischem Einschlag. Rassenschandeverfahren galten grundsätzlich als politische Verfahren. Wenn versucht wird, das Kernproblem der NS-Justiz am Beispiel der Hamburger Rassenschande-Judikatur aufzuzeigen, so geschieht es - bei aller Vorsicht gegenüber Verallgemeinerungen -, weil sie prototypische Züge der Rechtsprechung in allen jenen Fällen trägt, die im Dritten Reich als politisch betrachtet wurden.

Anfang Dezember 1935 hatte der Oberstaatsanwalt beim Landgericht Hamburg die Einrichtung eines Sondergerichts für Strafverfahren bezüglich des Blutschutzgesetzes angeregt, um eine einheitliche Rechtsprechung und rasche „,Klärung verschiedener Zweifelsfragen des Gesetzes“" zu erzielen. ${ }^{1}$ Dieser Anregung folgte das Landgerichtspräsidium durch den Beschluß, ,die Strafverfahren gegen das Gesetz zum Schutz des deutschen Blutes und der deutschen Ehre vom 15. September 1935 der Großen Strafkammer VI zuzuweisen". ${ }^{2}$ Zweifellos konnte dadurch die Einheitlichkeit der Rechtsprechung auf diesem besonderen Gebiet besser gewahrt werden. Doch wirkt eine solche Abspaltung von der allgemeinen Rechtspflege auch in ganz anderen Richtungen. Es bildet sich eine neue Spezialistengruppe, die für ihr Sonderreferat bestimmte Rechts- und Sachkriterien entwickelt.

${ }^{1}$ Schreiben des Oberstaatsanwalts an den Präsidenten des Landgerichts in Hamburg, 4. 12. 1935.

(Kopie im Besitz des Verfassers)

2 Undatierte Mitteilung des Landgerichtspräsidiums an den Oberstaatsanwalt. 
Diese Kriterien treten mehr und mehr an die Stelle der allgemeinen Rechtsgrundsätze und bergen für die Spezialisten auch die Gefahr einer Rechtsentfremdung. Eine solche Entwicklung, begünstigt durch einschlägige Weisungen, war in der Rechtsprechung der Hamburger 6. Strafkammer tatsächlich festzustellen. Wie wenig die Begriffswelt, die aus der speziellen Rassenschande-Judikatur entstand, schließlich von Rechtsüberlegungen bestimmt wurde, zeigt die Veränderung des Begriffs "Geschlechtsverkehr". ${ }^{3}$

Die Rechtsprechung ging, wie an anderer Stelle ausgeführt, über den vollzogenen Geschlechtsverkehr weit hinaus. Die unter dem biologischen Gesichtspunkt unbedenklichen Handlungen wurden als Ersatzhandlungen verurteilt und brauchten nicht mehr mit dem „Schutze der deutschen Ehre“ begründet zu werden. Doch ermöglichten die in die Bezeichnung des Gesetzes einbezogenen Wörter vom Schutz „der deutschen Ehre“ die Unterbindung jeder sexuellen Beziehung zwischen Juden und Nichtjuden, unabhängig von der sozialen Stellung und moralischen Qualität der Beteiligten. So war auch der Verkehr von Juden mit Prostituierten strafbar. ${ }^{4}$

Wenn das Reichsjustizministerium im Spätsommer 1936 die Einrichtung von Sonderstrafkammern, vielleicht nach dem Hamburger Vorbild, veranlaßte, wurde daher weit mehr als eine Vereinheitlichung der Rechtsprechung erreicht, nämlich eine spezialisierte Rassenschande-Judikatur, die zu einer weiteren extensiven Interpretation des Gesetzes führte. Auch wurde die Beeinflussung der Rechtsprechung in Rassenschandeverfahren erleichtert, wenn man in jedem Landgerichtsbezirk jeweils nur mit einem einzigen Kammervorsitzenden zu tun hatte. Ebenso stiegen die Chancen, diese Kammern nach und nach mit politisch ,zuverlässigen“ Vorsitzenden zu besetzen.

Das Reichsjustizministerium hatte die Rechtsprechung in Rassenschandeverfahren von Anfang an auf das Genaueste verfolgt. ${ }^{5}$ Am 17.12.1935 wurde allen Gerichten auferlegt, dem Reichsjustizministerium über Rassenschandefälle zu berichten. ${ }^{6}$ Ein Vierteljahr nach dieser Weisung erging an alle Generalstaatsanwälte und Oberstaatsanwälte ein Rundschreiben über die Handhabung des Blutschutzgesetzes. Unter Bezugnahme auf das Schreiben an den Oberstaatsanwalt in Stuttgart ${ }^{7}$ verwies das Reichsjustizministerium ergänzend auf folgendes:

„(1) §5 Abs. 2 des Gesetzes zum Schutze des deutschen Blutes und der deutschen Ehre droht bei verbotenem außerehelichem Geschlechtsverkehr zwischen Deutschen und Juden wahlweise Gefängnis oder Zuchthaus an. Nach den im Reichsjustizministerium getroffenen Feststellungen sind bisher regelmäßig Gefängnisstrafen bean-

3 Zur Erweiterung des Begriffs vgl. Kapitel 2.

${ }^{4}$ Stuckart und Globke schließen dies daraus, daß das Gesetz ,innerhalb seines Anwendungsgebiets uneingeschränkt" gilt. Stuckart-Globke, a. a. O., S. 112.

5 Auch andere Ministerien waren an der Wirkung der Rassenschande-Gesetzgebung interessiert. Eine entsprechende Anfrage des Reichspropagandaministers Goebbels wurde am 22.9.1937 vom Präsidenten des Hanseatischen Oberlandesgerichts an den Hamburger Landgerichtspräsidenten weitergeleitet und von diesem am 28.9.1937 beantwortet.

${ }^{6}$ Richtlinien für das Strafverfahren. Allgemeine Verfügung des Reichsministers der Justiz vom 13. April 1935 (Amtl. Sonderveröffentlichungen der Deutschen Justiz Nr.7) Anhang: Deckblätter zu den Richtlinien für das Strafverfahren und zu den Mitteilungen in Strafsachen, 1. Lieferung, enthaltend AV v. 7.8., 14.11. u. 17.12. 1935, Berlin o. J., S. 13-14.

7 Vgl. Kapitel 2. 
tragt und erkannt worden. Die Schwere der Vergehen gegen das deutsche Blut und die deutsche Ehre verlangt aber, daß - zumal nachdem nach Inkrafttreten des Gesetzes eine gewisse Zeit verstrichen ist - die Strafverfolgungsbehörden sich mit allem Nachdruck für die unbedingte Durchsetzung des Gesetzes, das für die Verwirklichung des nationalsozialistischen Rassegedankens von entscheidender Bedeutung ist, einsetzen und zu diesem Zwecke die Durchschnittsfälle unbedingt als schwere Fälle ansprechen, zumal hier in aller Regel Hartnäckigkeit oder offenbare Widersetzlichkeit gegen die Forderungen des nationalsozialistischen Rasseschutzes vorliegt; Zuchthaus ist daher in solchen Fällen die angemessene Strafe.

(2) Besonders unangemessen ist es, wenn jüdische Eingriffe in die Reinheit des deutschen Blutes im Endergebnis dadurch durch die Strafrechtspflege minder schwer geahndet werden, daß bei deutschen Rasseschändern ihr Deutschtum als strafschärfend hervorgehoben wird, daß das dann zu Zuchthausstrafen führt, während der Jude im entsprechenden Falle womöglich mit einer Gefängnisstrafe davonkommt.

(3) Schließlich erscheint es mir nicht angängig, wenn als Strafmilderungsgrund angeführt wird, es hätten zwischen dem Täter und dem anderen Teil schon seit längerer Zeit vor dem Inkrafttreten des Gesetzes geschlechtliche Beziehungen bestanden. Da das Gesetz die Fortsetzung solcher Beziehungen unbedingt verbietet, geht es nicht an, Verstöße gegen das Verbot mit dieser Begründung milder anzusehen.

Ich ersuche, bei den Anträgen zum Strafmaß diese Gesichtspunkte mit Nachdruck zu vertreten.

$$
\begin{aligned}
& \text { In Vertretung } \\
& \text { gez. Dr. Freisler." }
\end{aligned}
$$

Diese Einflußnahme auf das Verhalten der Strafverfolgungsbehörden blieb formell noch im Rahmen der bestehenden Rechtsordnung. Jedoch mußten die Argumente für die regelmäßige Beantragung der Zuchthausstrafe, wenn die Staatsanwaltschaften sich konsequent an die Weisung hielten, auch die Richter beeinflussen. Wollte eine Strafkammer nämlich von solchen Anträgen abweichend urteilen, mußte sie in jedem einzelnen Fall mit einem Revisionsantrag der Staatsanwaltschaft rechnen und zudem riskieren, vom Reichsgericht zurechtgewiesen zu werden. Das nächste vorliegende Rundschreiben des Reichsjustizministeriums datiert vom 1.9.1936 und lautet:

„Betrifft Handhabung des Gesetzes zum Schutze des deutschen Blutes und der deutschen Ehre vom 15. September 1935.

Bei der Überwachung der Rechtsprechung in den Verfahren wegen Zuwiderhandlung gegen die $\S \S 2,5$ Abs. 2 des Blutschutzgesetzes ist es aufgefallen, daß die von den Gerichten verhängten Strafen auch bei gleichgelagerten Fällen im Strafmaß außerordentliche Unterschiede aufweisen, und daß weiter einzelne Strafkammern es trotz der Ausführungen in der RV v. 2. März (April) 1936 - 1121 IIa 18501/36 - anscheinend grundsätzlich vermeiden, auch in schweren Fällen auf die angebrachte Zuchthausstrafe zu erkennen. Es ist z. B. unverständlich, wenn ein Gericht gegen einen Volljuden, der einem ihm vertrauenden Mädchen sich als Arier ausdrücklich

${ }^{8}$ Schreiben vom 2.4.1936, L.G 112, Bl. 7. 
ausgibt und es dadurch zum Beischlaf verleitet, entgegen dem zutreffenden Zuchthausantrag des Staatsanwalts nur auf 8 Monate Gefängnis erkennt.

Um diesem Mißstand abzuhelfen und zu einer einheitlichen sachentsprechenden Rechtsprechung zu gelangen, ersuche ich im Benehmen mit den Landgerichtspräsidenten auf die ihnen unterstellten Richter in geeigneter Weise nachdrücklich einzuwirken, damit einem Rasseverfall des deutschen Volkes auch von den deutschen Gerichten durch strenge Strafen entgegengewirkt wird. Eine solche Rechtsprechung wird sich unter anderem auch dadurch gewährleisten lassen, daß sofort die Verfahren wegen Verbrechens gegen das Gesetz vom 15. September 1935 einer einzigen Strafkammer (etwa einer bereits mit der Aburteilung von Sittlichkeitsverbrechen befaßten) zugeteilt werden. Ich ersuche auch insoweit, das Erforderliche beschleunigt zu veranlassen.

In Vertretung gez. Dr. Freisler" ${ }^{\circ}$

Hier wurde die Unabhängigkeit des Richters auch formell nicht mehr gewahrt, sondern die Rechtsprechung unmittelbar beeinflußt. Der Landgerichtspräsident in Hamburg berichtete am 15.9.1936 dem Oberlandesgerichtspräsidenten, daß er die vom Reichsjustizministerium ,geltend gemachten Gesichtspunkte schon aus früherem Anlaß, und jetzt wiederholt mit dem Herrn Vorsitzenden der Großen Strafkammer 6 besprochen habe".

Ein spezieller Eingriff in die hamburgischen Verhältnisse war das Schreiben des Reichsjustizministeriums vom 11.10.1937 an den Oberstaatsanwalt in Hamburg:

„Betr. Verschiedene Strafsachen wegen Rassenschande. Bei Durchsicht der Rassenschandeurteile ist in letzter Zeit aufgefallen, daß die 6. große Strafkammer des Landgerichts Hamburg in Strafsachen gegen Juden auffallend milde Urteile gefällt und fast nur auf Gefängnisstrafen erkannt hat. Auf der anderen Seite hat die gleiche Strafkammer am 20. und 25. August 1937 gegen zwei Arier Zuchthausstrafen von je zwei Jahren ausgesprochen. Es handelt sich namentlich um die folgenden Verfahren. ... [Folgen zwölf Fälle] ...

Ich ersuche Sie, Herr Oberstaatsanwalt, der Rechtsprechung der 6. Strafkammer in Rassenschandesachen Ihre besondere Aufmerksamkeit zuzuwenden.

Zugleich ersuche ich um Mitteilung, welche Strafanträge von den Sitzungsvertretern der Staatsanwaltschaft in den oben erwähnten Strafsachen gestellt worden sind."10

Diese Mitteilung machte der Oberstaatsanwalt am 21.10.1937 in Form einer Gegenüberstellung der beantragten und erkannten Strafen und erklärte am Schluß, daß ,die Dezernenten nochmals darauf hingewiesen worden (sind), nur ganz ausnahmsweise davon abzugehen, Zuchthausstrafen zu beantragen“. Dieser Brief an das Justizministerium ist dem Dezernatsleiter, dem Staatsanwalt Dr. R., und dessen Vorgesetzten vorgelegt worden, und der Oberstaatsanwalt Dr. J. hat das Schreiben bei allen Sachbearbeitern kursieren und abzeichnen lassen. Kurz darauf hatte sich auch der Präsident des Hanseatischen Oberlandesgerichts in die Angelegenheit eingeschaltet und am

\footnotetext{
${ }^{9}$ Rundschreiben des Reichsjustizministeriums an alle Oberlandesgerichtspräsidenten, nachrichtlich an alle Generalstaatsanwälte, LG 112, Bl. 6.

10 OStA Hbg, 1120.
} 
28. 10. 1937 einen Bericht angefordert. In diesem Bericht vom 5.11.1937 nahm Landgerichtspräsident K. zu den einzelnen Fällen ausführlich Stellung. Die Angriffe auf die Strafkammer wies er in jedem Punkt zurück. So heißt es auf Seite 4 des Berichts, „daß sich die von der Großen Strafkammer 6 erkannten Gefängnisstrafen gegen Juden durchaus vertreten lassen und zu Beanstandungen keinen hinreichenden Anlaß bieten“. ${ }^{11}$ Auf Seite 6 erklärte Dr.K., seiner „Überzeugung nach [ist] die Verhängung einer Zuchthausstrafe gegen die Arier vertretbar und gibt zu einem Eingreifen meinerseits keinen Anlaß".12

Die Sache war damit aber noch nicht beendet. OLG-Präsident Dr. Rothenberger reagierte am 11.11.1937 auf diesen Bericht mit dem nachstehend zitierten Schreiben:

„Die Durchsicht der mir übersandten Urteile, die anbei zurückgereicht werden, gibt mir Veranlassung, auf folgende Gesichtspunkte hinzuweisen, die m. E. grundsätzlich bei der Behandlung von Rassenschandesachen in den Vordergrund gerückt werden sollten:

1. Die Berücksichtigung persönlicher Verhältnisse oder besonderer Umstände sollte als Milderungsgrund nur nach sorgfältiger Prüfung der Frage erfolgen, ob die überragende staatspolitische Bedeutung der Rasseschutzgesetze und die Notwendigkeit ihrer strengen Durchführung gerade nach der Übergangszeit eine solche Berücksichtigung zulassen. Andernfalls könnte die Befürchtung gerechtfertigt sein, daß durch zu tiefes Eingehen auf die Umstände des einzelnen Falles und die persönlichen Verhältnisse des Täters der große Grundgedanke des Gesetzes verwässert wird und die unbedingt nötige rücksichtslose Bekämpfung der Rassenschande nicht mit der erforderlichen Energie erfolgt.

2. Es würde mir zweckmäßig erscheinen, wenn in den Urteilsbegründungen die persönlich-menschlichen Beziehungen zwischen den an der Rassenschande Beteiligten mit einiger Zurückhaltung behandelt würden. Nach der Auffassung des Gesetzgebers ist das Verbrechen der Rassenschande ein so schwerer Verstoß gegen Grundgesetze, die zur Erhaltung des Volkes erforderlich sind, daß demgegenüber die Berücksichtigung menschlicher Gesichtspunkte erst nach sorgfältiger Prüfung erfolgen darf. Es wird dem unverbildeten gesunden Volksempfinden schwer verständlich sein, wenn in solchen Urteilen allzu sehr etwa das Menschlich-Tragische oder die innigen persönlichen Beziehungen u.dgl. hervorgehoben werden.

Ich bitte, Gelegenheit zu nehmen, in einer Ihnen geeignet erscheinenden Weise die Richter, die in Rassenschandesachen eingesetzt werden, mit diesen Gesichtspunkten bekannt zu machen. Dabei weise ich noch auf das von dem Herrn Reichsminister der Justiz dem Herrn Oberstaatsanwalt in Hamburg gegenüber beanstandete Urteil in der Strafsache gegen W. B. - 11 KLs 63/37-66/37 - hin. In diesem Urteil hat das Gericht in den Strafzumessungsgründen ,zu Gunsten' des Angeklagten als richtig unterstellt, daß er die ernsthafte Absicht gehabt habe, die Jüdin ${ }^{13}$ zu heiraten. Auch hier wird eine offenbar tiefere menschliche Beziehung und ernsthafte Zuneigung in

11 LG 112, Bl. 22.

12 Ebenda, Bl. 24.

${ }^{13}$ Diktatfehler, muß heißen „Nichtjüdin“" oder „Arierin“. 
Verbindung mit der ernsten Absicht, die Zeugin zu heiraten, als Milderungsgrund aufgeführt. Mit Recht verweist der Herr Reichsminister der Justiz hierzu auf das Urteil des Reichsgerichts vom 31. Mai 1937 - DJ S. 1039 - und die in dieser Entscheidung zum Ausdruck gekommene Rechtsauffassung. “14

Dr. K. hatte auf der zweiten Seite dieses Schreibens vermerkt, daß der Vorsitzende der Strafkammer 6 von der Auffassung des Hanseatischen Oberlandesgerichtspräsidenten verständigt wurde, und hinzugefügt: „Sie entspricht vollen Umfangs seinen Ansichten." $" 15$

Mit jenem Schreiben hatte Oberlandesgerichtspräsident Rothenberger begonnen, den Verfahren vor der Hamburger 6. Strafkammer sein besonderes Augenmerk zuzuwenden. Dieser Mann, der seit 1933 an der Spitze der Hamburger Justizverwaltung stand, war ein äußerst begabter Jurist voller Schaffenskraft und Vitalität, zugleich ein unbedingter Nationalsozialist. Er gehörte zu den Juristen, die, wie er in einem Vortrag formulierte, ,gefühlsmäßig sich dem Nationalsozialismus mit Haut und Haar verschrieben" hatten. ${ }^{16}$ Obschon er gewisse romantische Vorstellungen von souveräner Rechtsprechung in der Art eines germanischen Volksrichters hegte, war er vor allem Realpolitiker, der wußte, daß weder seine nationalsozialistischen Anschauungen noch seine Rechtspflege-Ideen verwirklicht werden konnten, solange die Justiz nicht völlig in Händen nationalsozialistischer Richter lag. So hatte er von Anfang an seine beachtliche Willenskraft dafür eingesetzt, die Justizverwaltung mit nationalsozialistischem Geist zu durchdringen und seinem Ziel einer ausschließlich nationalsozialistischen Richterschaft so nahe wie möglich zu kommen.

Dem hamburgischen Justizverwaltungsblatt des Jahres 1933 ist zu entnehmen, mit welcher Linientreue Dr. Rothenberger vorging. In einem Erlaß vom 24.6. 1933 über die „Bekämpfung der Herstellung und Verbreitung illegaler Druckschriften“ beauftragte er die Staatsanwaltschaft, , die Gerichte immer von neuem darauf hinzuweisen ..., daß nur durch schwere abschreckende Strafen das . . volksschädliche Treiben der marxistischen und kommunistischen Hetzer gebrochen werden kann“" ${ }^{17}$

In zwei Erlassen vom 22. Juli 1933 über die „Anschaffung nationalsozialistischen Schrifttums“ und über die „Erziehung zum nationalsozialistischen Staatsgedanken und Zulassung von Aushängen politischen Inhalts in den Diensträumen“ wird die Pflicht

${ }^{14}$ LG 40 E - 1a (6).

15 Wenn auch das Eintreten des Landgerichtspräsidenten für die Urteile der Strafkammer zweifellos deren Vorsitzenden und ständigen Beisitzern eine fühlbare Stütze war, so beweist der gesamte Schriftwechsel und besonders die Tonart der Briefe des Reichsjustizministeriums und des Oberlandesgerichtspräsidenten, wie wenig die Unabhängigkeit der Rechtsprechung innerlich von den Dienstvorgesetzten der Richter anerkannt wurde. Die scharfe und mißtrauische Kritik und die Uberwachung der Urteile mußte bei den betreffenden Richtern das Gefühl wecken, daß3 sie sich für das, was ihnen Rechtens zukam und wozu ihre Pflicht sie nötigte, nämlich für ihre unabhängige Urteilspraxis, gerade denjenigen Stellen gegenüber zu rechtfertigen hatten, die für ihr Dienstfortkommen entscheidend waren. Die fallweise mögliche Auswirkung dieses Gefühls auf die Rassenschande-Judikatur ist evident.

${ }^{16}$ Curt Rothenberger, Nationalsozialistisches Rechtsdenken und einheitliche deutsche Justiz, Hamburg 1934, S. 17.

17 Hamburgisches Justizverwaltungsblatt, 22 (1933), S. 33. 
der Behördenleiter betont, ,die Beamten zu eingehender Beschäftigung mit dem Gedankengut des Nationalsozialismus“ anzuhalten und mit den "Grundsätzen der neuen Staatsauffassung bekanntzumachen“. ${ }^{18}$ „Ohne gründlichste Beherrschung des nationalsozialistischen Staatsgedankens" könnten Beamte und Angestellte ,in vielen Fällen sachdienliche Entscheidungen überhaupt nicht treffen . . . Erforderlich ist in erster Linie die möglichst rege Beteiligung an den öffentlichen Kundgebungen, Versammlungen und sonstigen (auch kleineren) Veranstaltungen der NSDAP., von denen sich bisher besonders die höhere Beamtenschaft in weitem Umfange zurückgehalten hat. . . . Der Nichtbesuch dieser Veranstaltungen wird als Unfreundlichkeit gegen den neuen nationalen Staat und als eine bewußte Ablehnung der nationalsozialistischen Bewegung angesehen. Wer nicht in den Verdacht verneinenden Verhaltens zu kommen wünscht, wird daher bestrebt sein, sich mehr als bisher nicht nur an den Veranstaltungen seiner Fachschaft, sondern auch an den sonstigen öffentlichen Veranstaltungen zu beteiligen", 19

Ein dritter Erlaß vom 28. Juli 1933 betrifft „Maßnahmen zur Bekämpfung der Sabotage des nationalsozialistischen Aufbaus“. „Fälle einer Auflehnung gegen den Führer oder einer Sabotage des nationalsozialistischen Aufbaus sind - nötigenfalls unter vorübergehender Zurückstellung weniger wichtiger Strafsachen - mit größter Energie und Schnelligkeit zu bearbeiten“. 20

Den gleichen Elan entwickelte nun das Hanseatische Oberlandesgericht bei den Rassenschandeverfahren. Am 31.1.1938 bat der Präsident des OLG darum, ihm ,künftig je eine Ausfertigung der in Rassenschande-Sachen ergehenden Urteile vorzulegen" ${ }^{21}$ Laut Notiz auf diesem Schreiben wurde am 2.2.1938 mit dieser Vorlage begonnen. Aus der Zeit von März bis November 1938 blieb eine ganze Reihe von Urteilen erhalten, die mit handschriftlichen Bemerkungen und Zeichen aus dem Präsidialbüro versehen sind und die Einstellung des OLG zur Tätigkeit der 6. Strafkammer zeigen. Erhalten blieben außerdem zwei Stellungnahmen, deren eine von Dr. Rothenberger unterzeichnet ist, während die andere zweifellos auf seine Anweisung zurückgeht.

In Sachen F 132 hatte Dr. Rothenberger am 28.3.1938 geschrieben:

„An den Herrn Landgerichtspräsidenten in Hamburg.

Betrifft: Urteile in Rassenschandesachen.

Bei der Durchsicht der Rassenschandeurteile ist das außerordentlich milde Strafmaß von 1 Jahr 6 Monaten Gefängnis in der Sache gegen H.D. - A. Z.: (36) 11 KLS. 10/38, 7/8, aufgefallen. Nach den Feststellungen der Strafkammer ist der Angeklagte am 20. September 1937 ausdrücklich von dem Zeugen K. vor einem Geschlechtsverkehr mit der S. gewarnt worden. Wenn der Angeklagte ungeachtet dieser Warnung ein weiteres Zusammentreffen mit dieser Zeugin verabredet hat und sich, als die Zeugin nicht erschien, von Alsterdorf mit dem Fahrrade zum Valentinskamp begeben hat, um doch noch einen Geschlechtsverkehr mit der Zeugin herbeizuführen, so

18 Ebenda, S. 38 (Erlaß vom 22.7.1933)

19 Ebenda, S. 38-39 (Erlaß vom 26.7.1933).

20 Ebenda, S. 39.

21 OLG $324-1 \mathrm{a} / 1$. 
liegt in diesem Verhalten ein ganz krasser und bewußter Verstoß gegen das Blutschutzgesetz. Bei dieser S9chlage konnte dem Angeklagten kaum zugute gehalten werden, daß er nur in einem einzigen Fall gegen das Gesetz verstoßen habe; vielmehr muß man aus seinem Verhalten wohl annehmen, daß auch der frühere Verkehr mit der S. nicht unterblieben wäre, selbst wenn der Angeklagte damals schon gewußt hätte, daß es sich bei der Zeugin um eine Jüdin handelt. Ob die Tatsache mildernd berücksichtigt werden konnte, daß dem Angeklagten nicht widerlegbar ist, daß er sich nach dem letzten Vorfall vom 24. September endgültig von der Zeugin hat trennen wollen, kann lediglich aus den Urteilsgründen nicht entnommen werden; für diese Frage müßte es wohl maßgeblich darauf ankommen, wann der Angeklagte verhaftet worden ist, ob er also überhaupt noch die Möglichkeit gehabt hat, weiterhin mit der Zeugin in Verbindung zu treten; andernfalls dürfte alles dafür sprechen, daß es sich dabei um eine Ausrede handelt.

gez. Dr. Rothenberger." 22

Am 20.5.1938 folgte ein Begleitschreiben des OLG zum retournierten Urteil F 146 mit der Randbemerkung: ,starke seelische Bindungen! - (es geht also wieder los mit der Unvorsichtigkeit)“. Dies letztere bezog sich auf die Rüge des Reichsjustizministeriums, nach welcher tiefere gefühlsmäßige Beziehungen nicht strafmildernd sein dürften, und es war daher ,unvorsichtig“, das nur auf Gefängnis lautende Urteil mit den beanstandeten Redewendungen zu begründen.

Auf dem Urteil vom 9.5.1938 gegen den Nichtjuden J. (F 151), das Ende Mai zum OLG kam, stehen zwei Notizen. Erstens: „,Übergangsfall‘ - (wieso?)“; zweitens sind aus dem Urteil die Worte zitiert: „menschlich verständlich“. Beides zeigt, daß dieses Urteil als zu milde angesehen wurde.

Ein Schriftstück vom 30.5. 1938 beweist, welcher Geist im OLG bei der Durchsicht der Urteile der Strafkammer 6 gewaltet hat.

\section{, Vermerk:}

Die Durchsicht der neuesten Rassenschandeurteile gibt hinsichtlich der sprachlichen Fassung zu einigen Bedenken Anlaß.

I.

In einer außerordentlich großen Anzahl der Urteile findet sich die schon fast stereotype Redewendung: das Blutschutzgesetz sei ein Grundgesetz des 3. Reiches; die Rassenschande sei daher exemplarisch zu bestrafen. Grundsätzlich könne nur Zuchthaus in Frage kommen, das Gericht habe aber diesen Fall als einen milden angesehen.

II.

In der Sache gegen B. 12/38 Seite 6 ist ausgeführt, das Gericht habe bei der Strafzumessung davon ausgehen müssen, daß es sich um einen schweren Fall handele. Diese

${ }^{22}$ OLG $3240 \mathrm{E}-\mathrm{L} 6 \mathrm{a}$ (F 132). 
Ausdrucksweise könnte einem Böswilligen Gelegenheit zur Kritik geben; man könnte die Sache so hinstellen, als ob das Gericht dieses „müssen“ bedaure.

III.

In der Sache gegen Sch. 22/38 Seite 6/7 und M. 20/38 Seite 4 ist zu Gunsten des Angeklagten berücksichtigt worden, „daß es sich um ein altes Verhältnis handele“. Nach der Rechtsprechung des Reichsgerichts kann diese Tatsache als strafmildernd nicht in Betracht gezogen werden.

IV.

In der Sache gegen A. 17/38 Seite 5 und 7 ist von „engen seelischen Bindungen“ die Rede, die zu einer milderen Beurteilung des Falles geführt haben. Außerdem ist als mildernd angesehen worden, daß der Angeklagte nach Holland habe auswandern wollen, um (?) auf diese Weise eine Trennung von der arischen Frau herbeizuführen. Das ist wohl nicht sehr wahrscheinlich, daß der Angeklagte gewissermaßen aus Achtung vor dem Blutschutzgesetz auswandern wollte; für diesen Entschluß dürften wohl andere Motive bei weitem ausschlaggebender gewesen sein. Da das rassenschänderische Verhältnis auf Seiten des Juden Ehebruch darstellte und nach der Feststellung des Gerichts 2 Jahre nach Erlaß des Gesetzes fortgesetzt ist (,und seit langen Jahren wie eine Ehe geführt worden ist"), erscheint es immerhin sehr fraglich, ob man noch von einem milden Fall sprechen konnte.

V.

In der Sache gegen F. 16/38 ist ohne weitere Erläuterung ausgeführt, daß der angeklagte Arier den Ehemann der volljüdischen Frau als seinen ,,Vorgesetzten im Luftschutzbund" kennengelernt habe. Aus der Sache 11/38 ergibt sich zwar, daß dieser Ehemann seinerseits Arier ist, das hätte man aber zweckmäßigerweise wohl auch in dem Urteil gegen F. kurz zum Ausdruck gebracht.

VI.

In der Sache gegen B. 12/38 Seite 2 können die Ausführungen über die Schuldverteilung im Ehescheidungsurteil dahin verstanden werden, daß die Strafkammer sich mit diesem jedenfalls nicht identifizieren wolle. Warum an dieser Stelle nicht kurz und bündig geschrieben worden ist, die Ehe sei im Jahre 1935 aus Verschulden des Angeklagten geschieden worden, ist nicht ganz ersichtlich.

\section{VII.}

Allgemein fällt die weitgehende Anrechnung der Untersuchungshaft auf, die ,aus Billigkeitsgründen" erfolgt. Worin diese Billigkeitsgründe erblickt werden, geht aus den Urteilen nicht hervor und ist einigermaßen unverständlich, wenn, wie in der Sache gegen Schm. 22/38 Seite 7, kurz vorher die Unaufrichtigkeit des Angeklagten im Vorverfahren und in der Hauptverhandlung ausdrücklich erwähnt wird. 


\section{VIII.}

Von den vorgelegten Urteilen (8) ist nur in 3 Fällen Zuchthaus verhängt worden, darunter in einer Sache wegen Beihilfe zur Rassenschande in Tateinheit mit schwerer Kuppelei.

Hamburg, den 30. Mai 1938.

gez. B. “23

Mitte Juli 1938 erhielt das OLG Kenntnis des Urteils vom 23.5.1938 gegen den nichtjüdischen Herrn Sch. (F 159). Der Angeklagte war freigesprochen worden, und auf der ersten Urteilsseite ist zu lesen „Arier - frei - nicht gerecht!“ Abermals protestierte das OLG gegen eine zu schonungsvolle Auslegung des Gesetzes. Gleiches geschah im Fall des jüdischen Herrn W. (F 188), der am 5.8.1938 zu einer zehnmonatigen Gefängnisstrafe verurteilt worden war. Auf dem Urteil stehen die mißbilligenden Stichworte: „Ersatzverkehr - Übergangsfall. Strafzumessung“.

Ende September kam die nächste Beanstandung. Hier handelte es sich um den jüdischen Angeklagten B. (F 190), der eine längere, seelisch tief begründete Beziehung zu einer nichtjüdischen Frau unterhalten hatte. B. war vorbestraft. Es war ein klarer Fall von Rassenschande. Der Staatsanwalt hatte drei Jahre Zuchthaus und drei Jahre Ehrverlust beantragt, die Kammer verurteilte B. zu drei Jahren Zuchthaus. Auf der ersten Seite des Urteils ist handschriftlich vermerkt: „Kriegsfreiw[illiger] Front verwundet EK II vorbestr[aft] Schieber 3 Jahre sehr wenig warum kein E[h]rverlust? Kein Verhältnis zu G. Frau [von] Epa entlassen, dann jude unterstützt.“

In diesem Fall hatte der Unmut des Kommentators sich sowohl gegen die Staatsanwaltschaft als auch die Strafkammer gerichtet. Nur gegen die Strafkammer richtete sich die Kritik des OLG am Freispruch des jüdischen Rechtsanwalt Dr. B. (F 191), obwohl die Staatsanwaltschaft eine Gefängnisstrafe von 15 Monaten beantragt hatte. Das groß hingeworfene und unterstrichene Wort „Frei“" mit Rufzeichen zeugt vom Ärger über diesen Fall.

Konsequent wurde im OLG auch die Tätigkeit eines beisitzenden Richters verfolgt, der häufig den Kammervorsitzenden vertrat. Aus einem Schreiben des Landgerichtspräsidenten vom 1.11.1938 geht hervor, daß einige Urteile, an denen Herr E. beteiligt war, dem Oberlandesgerichtspräsidium gesondert zugeschickt wurden, darunter das Urteil im Fall 162. Auf der ersten Seite des Urteils zeigt ein dicker Blaustiftpfeil auf den Namen des damals vorsitzenden Richters E. Unter der Notiz des Strafantrags ,2 Jahre Zuchthaus“" steht: „Über Antrag“. Der deutschblütige Angeklagte war nämlich zu drei Jahren Zuchthaus und drei Jahren Ehrverlust verurteilt worden. Links unten auf der Seite stehen die Buchstaben KZ und darüber ein Hinweis auf die Seiten 14 und 15 des Urteils.

Der Unwille des OLG war in diesem Fall wohl nicht nur durch das harte Urteil gegen einen Nichtjuden ausgelöst, sondern durch die zweifellos höchst unvorsichtige Formulierung im Zusammenhang mit dem angeblichen Erpressungsversuch der Zeugin W. Im Urteil wurde eine Erpressung als unwahrscheinlich hingestellt. Zwar sei sie der jüdi-

${ }^{23}$ OLG 400 (1a) (2). 
schen Zeugin an sich zuzutrauen, aber diese sei andererseits intelligent genug, um zu wissen, daß sie dann vermutlich in ein $\mathrm{KZ}$ gekommen wäre! Diese Stelle ist dick unterstrichen und am Rande mit einem dicken Ausrufungszeichen und drei Blaustiftstrichen versehen.

In den Akten befindet sich dazu ein Vermerk vom 5.11.1938, der von Herrn B. abgezeichnet wurde: „Die anliegenden Urteile habe ich heute Herrn Präsidenten L. vorgetragen. Es ergibt sich daraus, daß Herr Dr. E. wiederholt als Vorsitzender gesessen hat. Irgendwelche Beanstandungen hinsichtlich der Urteile sind nicht zu erheben." ${ }^{24}$

Das ist eine unklare Formulierung. Beanstandungen waren ja erhoben worden! Man hatte bei der Úberprüfung der Urteile und Begründungen aber offenbar festgestellt, daß Dr.E. sachlich kein Vorwurf zu machen war. Da alle beteiligten Richter wußten, daß ihre Urteile auch an den OLG-Präsidenten gingen, dessen dauernde Kritik man verspürte und dessen Einstellung auch ihre Beförderungschancen beeinflußte, kann diese Praxis kaum wirkungslos geblieben sein. Rothenberger war jedoch nicht der Mann, der sich darauf beschränkte.

Die Nadelstich-Taktik fortgesetzter kritischer Anmerkungen und die Kontrolle durch übergeordnete Dienststellen konnten das Sicherheitsgefühl der Richter wohl untergraben, doch keine Änderung herbeiführen. Die Unabsetzbarkeit und Unversetzbarkeit der Richter war ein schweres Hemmnis auf dem Wege Rothenbergers zu seinem Ziel einer nationalsozialistischen Richterschaft. Es ist daher charakteristisch, daß sich Rothenberger bereits wenige Monate nach seinem Amtsantritt mit der nationalsozialistischen Schulung der Referendare beschäftigte. Unter dem Datum des 19. 9. 1933 erschien im Hamburgischen Justizverwaltungsblatt ein Erlaß über das „Lagerleben der zur zweiten juristischen Prüfung zugelassenen Referendare". Nach der Feststellung, daß die Stellung als Richter, Staatsanwalt, Verwaltungsbeamter, Rechtsanwalt und Notar im nationalsozialistischen Staat ,charakterliche Eignung zum Diener der Volksgemeinschaft" voraussetze, heißt es:

„Diese Eignung muß im Zusammenleben mit anderen Volksgenossen erprobt werden, damit entschieden werden kann, ob der Referendar nicht nur intellektuell, sondern auch charakterlich geeignet ist, wichtigste Hoheitsaufgaben des Staates zu erfüllen.

Ich ordne deshalb im Einvernehmen mit den Justizverwaltungen von Bremen und Lübeck an:

1. Im Rahmen der zweiten juristischen Prüfung hat jeder arische männliche Referendar nach Absolvierung der schriftlichen Arbeit einen Zeitraum von etwa 8 Wochen in einem Lager in Gemeinschaft mit anderen Referendaren und Arbeitsdienstfreiwilligen zu verbringen. Die Teilnahme an dem Lagerleben ist Dienst und Bedingung der Prüfung, soweit nicht durch amtsärztliches Attest die Ungeeignetheit des Referendars festgestellt wird.

2. Das Lagerleben ist so zu gestalten, daß es Gelegenheit gibt, die charakterliche

${ }^{24}$ OLG 3240 E - L 6a, Anlage. 
Eignung des Referendars zu erproben. Durch Teilnahme am Arbeitsdienst in Gemeinschaft mit Volksgenossen aller Stände soll festgestellt werden, ob der Referendar Sinn für Kameradschaft und Volksverbundenheit hat.

3. Der bestellte Führer ist Vorgesetzter der Referendare und verantwortlich für die Durchführung des Lagerlebens nach den oben bestimmten Richtlinien. Er hat nach Beendigung des Lagerlebens eine schriftliche Äußerung darüber abzugeben, wie sich der Referendar während der Zeit des Lagerlebens geführt hat und welche charakterlichen Eigenschaften sich bei ihm besonders gezeigt haben. Er ist Mitglied der Prüfungskommission. “25

Dieser Erlaß wurde dann inhaltlich auch in die Prüfungsordnung der Hanseatischen Prüfungskommission übernommen.

In einem weiteren Erlaß vom 2. Dezember 1933 hatte Herr Dr. Rothenberger einen Schulungskurs ,für die älteren Referendare im Anschluß an die Arbeiten der politischen Fachgemeinschaft der Universität" angekündigt. In diesem Kursus sprachen Dozenten über die Ideenwelt des Nationalsozialismus.

„Zur Teilnahme verpflichtet sind die Referendare, welche eine Aufforderung von der Landesjustizverwaltung erhalten. Die Teilnahme an dem Kursus gehört zur Berufsausbildung, ist also Dienst. Sie geht daher jedem anderen Dienst vor. Eine Entschuldigung wird nur in den allerdringendsten Fällen zugelassen werden." 26

Auch Spezialkurse über Rassenfragen wurden durch Rothenberger gefördert. Die Kurse waren zumeist ebenfalls obligatorisch, um eine Nichtbeteiligung zu verhindern. Gelegentlich wurde die Teilnahme mittels finanzieller Pression erzwungen. So wurden alle Referendare, die Unterhaltszuschüsse empfingen und in der NS-Bewegung nicht aktiv tätig waren, durch ein Rundschreiben vom 8.11.1936 informiert:

„Nach § 4 der Allgemeinen Verfügung des Herrn RMI vom 16.5.1935 dürfen Unterhaltszuschüsse nur an solche Referendare bewilligt werden, die nach ihrer gesamten Persönlichkeit die Gewähr dafür bieten, daß sie auch den erhöhten Anforderungen voll entsprechen, die der Staat an seine Beamten stellt.

Ich fordere Sie deshalb auf, mir bis zum 16.12.1936 mitzuteilen, in welcher Weise Sie sich aktiv für die nationalsozialistische Bewegung betätigen oder aus welchen Gründen Sie eine solche Betätigung unterlassen. “27

Die Personalakte des damaligen Referendars enthält auch eine Liste, derzufolge dieses Schreiben an siebzehn Referendare ging. Dreien von ihnen wurde der Zuschuß völlig gestrichen und einem von monatlich 150 auf 100 Reichsmark gekürzt.

Bewerber um eine Anstellung oder Beförderung im Justizdienst mußten eine Art Unbedenklichkeitszeugnis der Parteiinstanzen vorlegen. Junge Juristen, die aus Gesinnungsgründen nicht ohnedies mindestens einer NS-Organisation angehörten, verschafften sich dieses politische Zeugnis durch ihren Eintritt in die Partei, SA oder SS. Die Zugehörigkeit zum nationalsozialistischen Juristenbund (später NSRB) wurde als selbstverständlich angesehen und reichte daher allein nicht aus, um als zuverlässig im

${ }^{25}$ Hamburgisches Justizverwaltungsblatt 22 (1933), S. 48.

${ }^{26}$ Ebenda, S. 68.

27 Personalakte des Referendars (des späteren Amtsgerichtsrats M.). 
nationalsozialistischen Sinne zu gelten. Da nun die jüngeren Juristen viel Zeit für Dienst in der Partei oder einer ihrer Gliederungen und für die Arbeiten der Fachschaft aufbringen mußten, hinderte sie allein schon Zeitmangel an der Festigung oder auch nur Wahrung ihrer geistigen Selbständigkeit. Ausnahmen hat es sicher gegeben, aber die vielen politisch Uninteressierten wurden zu einem unbewußten politischen Reflexverhalten im Sinne des herrschenden Systems veranlaßt.

Dr. Rothenberger förderte die jungen Juristen. Er gab ihnen das Gefühl, sich für sie persönlich zu interessieren, und setzte sich auch für das berufliche Fortkommen jüngerer Assessoren, Richter und Staatsanwälte ein, selbst wenn es gelegentlich Personen waren, deren fachliche Qualifikation zu wünschen übrig ließ. Es waren dies aber junge Männer, über die aus dem Referendarlager, aus der Partei oder der SA günstige Berichte vorlagen und die er aus der politischen Arbeit kannte.

Geht man der Frage nach, welchen Einfluß diese Personalpolitik auf die Zusammensetzung der Hamburger 6. Strafkammer hatte, so verfügt man über zwei Anhaltspunkte: das Alter der an der Kammer hauptsächlich tätigen Richter und, 1939, den Wechsel im Vorsitz der Kammer.

Unter den erhaltenen Urteilen in Rassenschandeverfahren stehen die Namen von 61 Richtern. Nur 18 dieser 61 waren an der Rassenschande-Judikatur in größerem Aus$\mathrm{ma} 3$ beteiligt. Die Untersuchung beschränkt sich auf diese 18 Richter.

Die nachstehende Tabelle zeigt die Anzahl der von jedem dieser 18 Richter abgeurteilten Personen, den Zeitpunkt des ersten und letzten bekannten Urteils sowie das Alter der vorsitzenden und beisitzenden Richter am Tag des ersten Verfahrens.

Tabelle 14 Übersicht über die hauptsächlich an Rassenschandeverfahren beteiligten Richter.

\begin{tabular}{|c|c|c|c|c|c|}
\hline Nr. & Richter & $\begin{array}{l}\text { Zahl d. } \\
\text { Verur- } \\
\text { teilten }\end{array}$ & $\begin{array}{ll} & \mathrm{Da} \\
\text { ersten } & \mathrm{Ve}\end{array}$ & $\begin{array}{l}\text { es } \\
\text { letzten } \\
\text { ns }\end{array}$ & $\begin{array}{l}\text { Alter am Tag d. } \\
\text { ersten Verfahrens }\end{array}$ \\
\hline 1 & $\mathrm{Da}$ & 167 & 12. 5.38 & 13. 4.43 & 27 \\
\hline 2 & $\mathrm{E}$ & 91 & 17. 8.36 & 13. 4.43 & 33 \\
\hline 3 & D & 88 & 8. 5.36 & 26. 5.39 & 48 \\
\hline 4 & W & 88 & 4. 1.39 & 13. 4.43 & 35 \\
\hline 5 & S-K & 53 & 8. 5.36 & 27. 4.38 & 27 \\
\hline 6 & $\mathbf{P}$ & 40 & 11.11 .36 & 27. 7.39 & 33 \\
\hline 7 & $\mathbf{M}$ & 39 & 16. 6.39 & 12. 2.40 & 27 \\
\hline 8 & Wa & 35 & 4. 1.39 & 24. 7.39 & 33 \\
\hline 9 & $\mathrm{H}$ & 29 & 8. 4.40 & 12. 2.42 & 51 \\
\hline 10 & Wu & 22 & 8. 5.36 & 27. 5.38 & 39 \\
\hline 11 & $\mathrm{St}$ & 21 & 1.11 .37 & 30.12 .38 & 30 \\
\hline 12 & $S-G$ & 19 & 5. 1.38 & 1.11.39 & 28 \\
\hline 13 & $\mathrm{Du}$ & 19 & 12. 4.40 & 12. 6.41 & 28 \\
\hline 14 & $\mathrm{C}$ & 15 & 2. 2.38 & 17.10 .38 & 34 \\
\hline 15 & Ho & 15 & 18. 9.39 & 1.11 .39 & 29 \\
\hline 16 & B & 12 & 11. 8.39 & 24.10 .39 & 52 \\
\hline 17 & $\mathrm{Et}$ & 9 & 27. 9.38 & 26. 9.39 & 35 \\
\hline 18 & $\mathrm{~L}$ & 9 & 30. 7.37 & 20. 7.38 & 46 \\
\hline
\end{tabular}


Von den in der Tabelle aufgeführten 18 Personen waren sechs als Assessoren tätig. Da aber Assessoren oft als Beisitzer auftraten, kann man ihre Mitwirkung nicht ignorieren. Von den an den Entscheidungen beteiligten Juristen waren demnach

$\begin{array}{ll}\text { zwischen 26 und 30 Jahre alt } & 5 \\ \text { zwischen 31 und 35 Jahre alt } & 3 \\ \text { zwischen 36 und 40 Jahre alt } & 5 \\ \text { zwischen 41 und 45 Jahre alt } & 1 \\ \text { zwischen 46 und 50 Jahre alt } & 1 \\ \text { zwischen 51 und 55 Jahre alt } & \underline{3} \\ & \underline{\underline{18}}\end{array}$

War diese Alterszusammensetzung der Strafkammer zufällig entstanden oder ein Ergebnis zielbewußter Personalpolitik? Vermutlich neigten die älteren LG-Räte dazu, eine Tätigkeit an anderen Kammern zu suchen, so daß an ihre Stelle Assessoren und jüngere Richter traten, die am Anfang ihrer Laufbahn und mindestens seit 1933 unter dem direkten Einfluß des Nationalsozialismus standen.

Der zweite Anhaltspunkt zur Frage nach dem Einfluß der Personalpolitik des OLG auf die Zusammensetzung der Hamburger 6. Kammer war der Wechsel im Vorsitz, der sich schon vor 1939 abzeichnete.

Am 17.11.1938 hatten die Teilnehmer an einer der regelmäßigen Präsidentenbesprechungen sich auch mit personellen Fragen beschäftigt. Das Protokoll dieser Besprechung enthielt folgenden Passus:

„,Landgerichtspräsident K. bat darum, dem LGDirektor Dr. . . die Strafkammer

6 (Rassenschandesachen) zu belassen, ihm allerdings einen besonders qualifizierten

Beisitzer zuzuteilen, etwa den LGRat ... vom Sondergericht. Senator Dr. Rothen-

berger stimmte $\mathrm{zu}$ und bat, LGRat ... auf jeden Fall der Strafkammer ... als

1. Beisitzer zuzuteilen. " 28

Anfang 1939 wurde dann schon über eine Versetzung des Landgerichtsdirektors diskutiert. Aus den Akten geht nicht hervor, wer sie schließlich veranlaßt hat, aber am 1.7.1939 wurde der Landgerichtsdirektor an das Oberlandesgericht Breslau versetzt. Der Vorsitz der 6. Strafkammer war frei, und bei der Neubesetzung fiel die Wahl auf den Landgerichtsrat W., der durch seine Tätigkeit am Sondergericht beim Oberlandesgerichtspräsidenten als besonders qualifiziert galt.

Der Landgerichtsdirektor war, als er nach Breslau ging, 51 Jahre alt, sein Nachfolger 35 Jahre.

Es ist für die gesamte Diskussion um die Unabhängigkeit der Richter von Interesse, an einem solchen Beispiel zu erkennen, welche Möglichkeiten eine äußerlich verfassungskonforme Justizverwaltung hat, die Gerichtsbarkeit zu beeinflussen. Eine andere Frage ist jedoch, ob Erfolge personalpolitischer Art sich in der Rechtsprechung niederschlagen.

Die nachstehende Tabelle gliedert die von der Hamburger 6. Kammer während der Jahre 1936 bis 1942/43 verhängten Gefängnis- und Zuchthausstrafen im Durchschnitt

${ }^{28}$ OLG $2200 \mathrm{E}-2 \mathrm{a} / 1, \mathrm{~S} .14$. 
nach Tagen und getrennt für jüdische und nichtjüdische Verurteilte. Sie zeigt, daß sich die Strafpraxis der Kammer von Anfang an verschärft hatte, bis 1939 der Höhepunkt erreicht wurde.

Tabelle 15 Durchschnittliche Strafdauer der verurteilten

\begin{tabular}{lllll}
\hline & Juden & \multicolumn{3}{c}{ Nichtjuden } \\
Jahr & Gefängnis & Zuchthaus & Gefängnis & Zuchthaus \\
\hline 1936 & 318 & 807 & 485 & 548 \\
1937 & 424 & 1066 & 430 & 889 \\
1938 & 585 & 1566 & 551 & 972 \\
1939 & 436 & 2213 & 573 & 852 \\
1940 & 641 & 1386 & 548 & 850 \\
1941 & 539 & 1422 & 356 & 943 \\
$1942 / 43$ & 730 & 1257 & 424 & - \\
\hline
\end{tabular}

Die Jahre sind zu 365 Tagen und die Monate mit 30 Tagen gerechnet.

Die Steigerung der Freiheitsstrafen entsprach der politischen Entwicklung, die immer härter gegen alle Gegner vorging. Scharfe Strafen gab es jedoch bereits von Anfang an, so daß man als Ergebnis sagen kann, daß die Beeinflussung durch Ministerium und OLG-Präsidenten sowie die Personalpolitik vielleicht zur Urteilspraxis beigetragen, sie aber nicht verursacht haben.

Die Staatsanwaltschaft als einer der stetigen und konsequent in derselben Richtung wirkenden Faktoren bei der Urteilsbildung der Richter trägt eine indirekte Verantwortung für den Ausgang der Rassenschandeverfahren. Direkt verantwortlich ist sie für das $\mathrm{Maß}$, in welchem die selbst im Dritten Reich vorhandenen Möglichkeiten zur Aufrechterhaltung einer gewissen Rechtsstaatlichkeit ausgenutzt wurden. Den beteiligten Beamten der Staatsanwaltschaft fällt es freilich leichter als den Richtern, sich von der persönlichen Verantwortung freizusprechen. Sie können sich darauf berufen, daß sie weisungsgebunden waren. ${ }^{29}$ Trotz aller Weisungen des Reichsjustizministeriums und - in Hamburg - aller Kontrollen durch den OLG-Präsidenten Rothenberger hatten die Staatsanwaltschaften jedoch eine nicht unerhebliche Ermessensfreiheit. Es lag oft im Ermessen der Staatsanwaltschaft, das Verfahren einzustellen, einen Tatbestand als Rassenschande, als versuchte Rassenschande oder als einen strafrechtlich belanglosen Vorfall anzusehen. Die Staatsanwaltschaft hat indes oft Anklage auch dann erhoben, wenn dies der Sachlage nach hätte vermieden werden können. Ein Beispiel ist der erwähnte Fall 296, in dem nichts vorlag, als daß der jüdische Beschuldigte sich von einer nichtjü-

29 In einigen Entnazifizierungsverfahren wurde derartiges vorgebracht. Es bezieht sich aber meistens nur darauf, daß man sich anständig betragen habe. Niemals wird behauptet, man habe einem jüdischen Angeklagten im Rahmen des Rechts geholfen. Eine Ausnahme war jedenfalls F 360. Nach schriftlicher Aussage des damaligen Angeklagten und nach einer dem Verfasser mündlich erteilten Auskunft hat der Staatsanwalt den Eid der Mutter des Angeklagten akzeptiert, obwohl er den starken Verdacht hatte, daß es sich um einen Meineid handelte. 
dischen Masseuse eine Magenmassage geben ließ. Ein anderes Beispiel ist der Fall 194, in welchem ein kastrierter Mann den Busen einer St. Pauli-Tänzerin gestreichelt und sie geküßt haben soll. Ein Gegenbeispiel ist jener deutschblütige Mann, der von der Polizei beschuldigt worden war, mit einer jüdischen Bürokollegin Rassenschande begangen zu haben. Er bestritt dies, worauf ihm vorgehalten wurde, er habe die Betreffende bei einem Betriebsausflug intensiv geküßt. Der Beschuldigte erwiderte: „Da muß ich aber schon sehr besoffen gewesen sein", worauf das Verfahren eingestellt wurde. ${ }^{30}$

Oft lag es auch im Ermessen der Staatsanwaltschaft, die Anklagepunkte zu vermehren oder nicht. Im Fall 348 z. B. hatte sie den Beschuldigten auch wegen versuchter Rassenschande angeklagt, weil er in Gegenwart einer Bekannten die Zeugin, die ihren neuen Pullover vorführte, unvermittelt an der Brust angefaßt habe. Daß das Gericht tatsächlich den Angeklagten auch deswegen mit einer Einsatzstrafe von einem Jahr Zuchthaus belegte, rechtfertigt nicht das Verhalten der Staatsanwaltschaft, die ganz überflüssigerweise diesen harmlosen Vorfall zu einem kriminellen Sachverhalt aufblies. Ähnliches gilt von der Ermessensfreiheit der Staatsanwaltschaft, Einzelfälle als fortgesetzte Handlung oder gesonderte Straftaten zu betrachten. Ein Beispiel für viele andere ist der Fall 235, in dem der Angeklagte zweimal innerhalb kurzer Zeit den Beischlaf mit derselben Frau vollzogen hatte, die Staatsanwaltschaft des ungeachtet Anklage wegen zweier Fälle von Rassenschande erhob und das Gericht den 32jährigen lungentuberkulösen Mann zu sechs Jahren Zuchthaus verurteilte.

Jeder Staatsanwalt hat die Möglichkeit objektiven Verhaltens, ist er nach dem Gesetz doch verpflichtet, sowohl die entlastenden Momente wie die belastenden Umstände geltend zu machen. Dieser Verpflichtung wurde jedoch bei den Rassenschandeverfahren selten genügt. Die Strafanträge waren insofern nicht objektiv, als sie oft in keinem Verhältnis zur Straftat standen. So wurde bei einem Angeklagten, dessen Beziehung zu einer nichtjüdischen Kollegin kein Vierteljahr gedauert hatte, eine Zuchthausstrafe von acht Jahren beantragt. ${ }^{31}$

Nicht objektiv war die Staatsanwaltschaft auch in anderer Hinsicht. Sie beantragte gegen jüdische Angeklagte zumeist Zuchthausstrafen, gegen nichtjüdische in Vergleichsfällen oft nur Gefängnisstrafen.

Viele Strafanträge waren überdies unsachlich, indem sie ohne Rücksicht auf den Stand des Verfahrens erfolgten. Dies wird vor allem an Fällen deutlich, in denen jüdische Angeklagte freigesprochen oder zu kürzeren Freiheitsstrafen verurteilt wurden. In der Verhandlung gegen Herrn $H$. stellte sich heraus, daß die von der angeblich beteiligten Frau erhobenen Beschuldigungen von ihr ausdrücklich vor ihrem Tode zurückgenommen worden waren. Für das eigentümliche Verhalten dieser Frau gab es eine zureichende Erklärung, die von anderen Zeugen eidlich gestützt wurde. Der Angeklagte hatte den besten Ruf, was auch im Gerichtssaal vorgebracht wurde. Er war in jeder Weise entlastet. Trotzdem wurde am 13.6.1938 eine Bestrafung des Angeklagten mit 21/2

${ }^{30}$ Persönliche Auskunft des Beschuldigten an den Verfasser.

31 F 246. Das Urteil lautete auf 7 Jahre Zuchthaus. Es hat zahlreiche Fälle ähnlich exzessiver Strafanträge gegeben, z. B. F 155, F 160, F 217, F 235, F 244 und F 303. 
Jahren Zuchthaus beantragt, wohingegen das Gericht freisprach. ${ }^{32}$ Dasselbe passierte dem gleichen Staatsanwalt am 6.3.1939. Der Angeklagte konnte einen klaren Beweis seiner Unschuld führen. Hier hätte es überhaupt nicht zu einer Anklage kommen dürfen. Aber völlig unberührt vom Ergebnis der Hauptverhandlung wurden 3 Jahre Zuchthaus beantragt. Urteil: Freispruch. ${ }^{33}$ Einer der Assessoren der Staatsanwaltschaft erlebte dies dreimal im Laufe von wenigen Monaten.

Am 15.6.1938 wurde gegen den deutschblütigen Herrn O. verhandelt, der seit 1932 Mitglied der NSDAP war. Herr O. hatte von 1931-1937 mit geringen Unterbrechungen ein Verhältnis mit einem etwa 20 Jahre jüngeren jüdischen Mädchen unterhalten. Längere Zeit hindurch hatte der Angeklagte die Zeugin ausgehalten. Als sie sich einmal getrennt hatten, schickte er ihr auf ihre Bitte sofort das Geld für die Fahrt von Königsberg nach Hamburg und begann das Verhältnis erneut. In diesem Fall hatte die Staatsanwaltschaft nicht etwa behauptet, es habe sich um mehrere getrennte Fälle von Rassenschande gehandelt, obwohl ja die Zusendung des Reisegeldes nach der Trennung ohne Zweifel ein selbständiger Entschluß war, das Verhältnis neu zu beginnen. Für diesen Fall fortgesetzter Rassenschande eines Parteigenossen von 1932 beantragte der Anklagevertreter $2^{1 / 2}$ Jahre Gefängnis. Das Gericht verurteilte Herrn O. zu drei Jahren Gefängnis. ${ }^{34}$

Wie weit war dieses Verhalten der Staatsanwaltschaft auf die NS-Politik und die vorgesetzten Dienststellen zurückzuführen und wie weit lag es an der Persönlichkeit der einzelnen Mitarbeiter des Dezernats? Bekannt sind die Namen von 21 Personen, die zwischen 1936 und 1943 im Rassenschande-Dezernat tätig waren. Nur acht von ihnen haben gegen zehn oder mehr Angeklagte plädiert. Und nur die hinsichtlich ihrer Einflußnahme wichtigsten Persönlichkeiten, der Dezernatsleiter Dr. R. und sein Mitarbeiter Staatsanwalt G., sollen näher ins Auge gefaßt werden.

Dr. R. wurde 1903 in einer kleinen Stadt geboren und war als Kind nach Hamburg gekommen. Seine Familie gehörte zum begüterten Mittelstand. Schon als Schüler hatte er sich rechtsradikalen Gruppierungen angeschlossen, dann einem Freikorps, der schwarzen Reichswehr und schließlich der Marine-SA, wo er es später zum Obersturmführer brachte. Ab 4.5.1932 wurde er Parteimitglied. Um die gleiche Zeit bestand er sein Staatsexamen und bemühte sich um Aufnahme in den Staatsdienst. Da die Bemühungen erfolglos waren, ließ er sich als Rechtsanwalt nieder.

Seine Beziehungen zu Hamburger Parteigrößen ermöglichten ihm, am 24.4.1933 als Regierungsrat beamtet zu werden. Er bekam einen verantwortungsvollen Verwaltungsposten, wurde aber nach knapp einem Jahr entfernt und am 1.4.1934 - ohne praktische Erfahrungen im Justizdienst - zum Staatsanwalt ernannt. Seine Ernennung scheint auf erheblichen Widerstand gestoßen zu sein, denn ein ihm vorgesetzter Staatsanwalt wurde verpflichtet, dem Reichsstatthalter regelmäßig über seine dienstliche Führung zu berichten. Erst nach längerer Zeit lauteten diese Berichte befriedigend. ${ }^{35}$

${ }^{32} \mathrm{~F} 176$.

${ }^{33} \mathrm{~F} 245$.

${ }^{34}$ F 177.

35 Personalakte Dr. R. 
Dr. R. war bei seinem Dienstbeginn in der Staatsanwaltschaft noch nicht 31 Jahre alt. Etwa Ende 1935 übernahm er die Bearbeitung der Rassenschandefälle, bald wurde er zum Dezernatsleiter und am 1.1.1938 zum Ersten Staatsanwalt befördert. In dieser Stellung sorgte er für äußerste Härte in der Verfolgung der Beschuldigten. Seine Tonart war schroff und verletzend, besonders, aber nicht nur, gegenüber jüdischen Angeklagten und Anwälten. Im Fall 88 bezeichnete er z. B. den nichtjüdischen Angeklagten Z. als einen ,typischen deutschen Intellektuellen, der noch nichts für Deutschland getan“ habe. Als Z. erwiderte, er habe am Ersten Weltkrieg teilgenommen, bemerkte Staatsanwalt R., er habe ,natürlich dieses Deutschland“, also das Dritte Reich, gemeint. ${ }^{36}$

Staatsanwalt G. war drei Jahre jünger als Dr. R., sein unmittelbarer Vorgesetzter. Bei der Machtübernahme war er noch Referendar. Am 1. Mai 1933, kurz nach bestandenem Examen, wurde er Mitglied der NSDAP, am 1. November 1933 SS-Mann. ${ }^{37} \mathrm{Er}$ avancierte zum Rottenführer und hat sich gelegentlich auch als Rechtsberater betätigt. In der Staatsanwaltschaft wurde er als Assessor zunächst der Hochverratsabteilung zugeteilt und 1937 zum Rassenschande-Dezernat versetzt. ${ }^{38}$ Soweit festzustellen ist, hat er vor der 6. Kammer die Anklage erstmals im Oktober 1937 vertreten. Den Eindruck, der für sein späteres Verhalten entscheidend wurde, gewann er offenbar im Verfahren gegen Herbert G., in dem er - es war sein vierter Fall - durchaus sachgemäß auf Freispruch mangels Beweises plädiert hatte. ${ }^{39}$ Die Kammer verurteilte den Angeklagten dennoch zu 3 Jahren Zuchthaus. Von da an war G. ein linientreuer Staatsanwalt. $^{40}$

Doch selbst unter dem Dezernatsleiter Dr. R. und seinem engsten Mitarbeiter Dr. G. verblieb den Anklagevertretern eine gewisse Möglichkeit, ihre abweichende Meinung zu äußern. Assessor Me., zum Beispiel, tat dies, indem er auch für die überwiegende Mehrheit der deutschblütigen Angeklagten Zuchthausstrafen beantragte - gewissermaBen nach dem Motto: Wenn gleiches Recht für alle unmöglich ist, verlange ich gleiches Unrecht für alle.

Die Zusammenarbeit der Staatsanwaltschaft mit der Kammer lag im Rahmen des Úblichen. Nur in wenigen Fällen ist die Kammer über die Anträge der Staatsanwaltschaft hinausgegangen, nämlich bei $9(7,6 \%)$ der jüdischen und bei $7(14,5 \%)$ der nichtjüdischen Angeklagten. Bei $82(68,9 \%)$ der jüdischen und $38(69,1 \%)$ der nichtjüdischen Angeklagten hat die Kammer den Strafantrag der Staatsanwaltschaft abgeschwächt. Bei den restlichen ca. $20 \%$ aller Fälle hat sie sich der Staatsanwaltschaft angeschlossen. Im Gegensatz zu den zahlreichen Revisionsanträgen der Verteidigung hat die Staatsanwalt-

${ }^{36}$ F 88, Auskunft des Herrn Z. an den Verfasser.

${ }^{37}$ Nach dem Krieg bekundete er, die SS bevorzugt zu haben, weil sie eine bessere Uniform trug und angesehener war als die SA.

${ }^{38}$ Personalakte Staatsanwalt G.

39 F 98. Siehe Kap. 6.

40 Auch bei der Staatsanwaltschaft ist das geringe Alter der an Rassenschandeverfahren beteiligten Mitarbeiter auffallend. Am 1.1.1940 war der Hamburger Dezernatsleiter 36 Jahre, sein Stellvertreter 33 Jahre alt. Von den sechs anderen Hauptbeteiligten war ein Staatsanwalt 37 Jahre, die fünf Assessoren waren im Alter zwischen 27 und 34 Jahren. 
schaft - soweit bekannt ist - nur gegen das auffallend milde Urteil der Kammer im Fall 360 Einspruch erhoben. Dies mag zum Teil daran gelegen haben, daß sich im Laufe der Jahre mehr und mehr die sogenannte außergerichtliche Urteilskorrektur durchgesetzt hat, d. h. durch polizeiliche Maßnahmen ausgeglichen wurde, was nach Ansicht der politischen Stellen von den Gerichten versäumt worden war.

Was aber bewog die Richter und Staatsanwälte, ihren Dienst in der Justiz als eine Funktion politischer Verfolgung aufzufassen? 